\title{
Survival After Lobectomy Versus Sublobar Resection for Stage IA Large-Cell Neuroendocrine Carcinoma of the Lung: A Population-Based Study
}

xiangyang yu ( $\square$ yuxy1991@yeah.net)

sun yat-sen university cancer center https://orcid.org/0000-0002-2372-8905

\section{Zirui Huang}

sun yat-sen university cancer center

\section{Mengqi Zhang}

Shenzhen Maternity and Child Healthcare Hospital

\section{Yongbin Lin}

sun yat-sen university cancer center

Rusi Zhang

sun yat-sen university cancer center

Yingsheng Wen

sun yat-sen university cancer center

\section{Xuewen Zhang}

sun yat-sen university cancer center

\section{Longjun Yang}

sun yat-sen university cancer center

\section{Dechang Zhao}

sun yat-sen university cancer center

\section{Lanjun Zhang}

sun yat-sen university cancer center

\section{Research article}

Keywords: pulmonary larger cell neuroendocrine carcinoma, SEER, sublobar resection, survival

Posted Date: April 5th, 2021

DOl: https://doi.org/10.21203/rs.3.rs-386977/v1

License: (1) This work is licensed under a Creative Commons Attribution 4.0 International License. Read Full License 


\section{Abstract}

Background: Due to the low incidence of pulmonary large cell neuroendocrine carcinoma (LCNEC), the survival analysis for comparing lobectomy and sublobar resection (SLR) for stage IA LCNEC remains scarce.

Methods: Patients diagnosed with pathological stage IA LCNEC between 1998 and 2016 were extracted from the Survenillance, Epidemiology, and End Results (SEER) database. The oncological outcomes were cancerspecific survival (CSS) and overall survival (OS). Kaplan-Meier analysis and Cox multivariate analysis were used to identify the independent prognostic factors for OS and CSS. Furthermore, propensity score matching (PSM) was performed between SLR and lobectomy to adjust the confounding factors.

Results: A total of 308 patients with stage IA LCNEC met the inclusion criteria: 229 patients $(74.4 \%)$ received lobectomy and 79 patients $(25.6 \%)$ received SLR. Patients who underwent SLR were older $(P<0.001)$, had smaller tumor size $(P=0.010)$ and fewer lymph nodes dissection $(P<0.001)$. The 5 -year CSS and OS rates were $56.5 \%$ and $42.9 \%$ for SLR, and $67.8 \%$ and $55.7 \%$ for lobectomy, respectively ( $P=0.037$ and 0.019 , respectively). However, multivariate analysis did not identify any differences between SLR group and lobectomy group in CSS $(P=0.135)$ and OS $(P=0.285)$; and the PSM also supported these results. In addition, age at diagnosis and laterality of tumor were identified as significant predictors for CSS and OS, whereas the number of lymph nodes dissection was a significant predictor for CSS.

Conclusions: Although SLR is not inferior to lobectomy in terms of oncological outcomes for patients with stage IA LCNEC, more lymph nodes can be dissected or sampled during lobectomy. Lobectomy should be considered as a standard procedure for patients with early-stage LCNEC who are able to withstand lobectomy.

\section{Background}

Pulmonary large cell neuroendocrine carcinoma (LCNEC), first reported in 1991, is a rare (around 3\% of all lung cancer) but aggressive subtype of lung tumors (5-year overall survival [OS] rate is only $16.2 \%$ ) [1-3]. On basis of the latest World Health Organization (WHO) classification of lung tumors, LCNEC is eliminated from the cluster of large cell carcinoma, and is regrouped into the high-grade neuroendocrine tumor (NET) along with small cell lung cancer (SCLC) [3]. LCNEC and SCLC share some histological features (including rosette, trabeculae, molding of nuclei and palisading, etc.), same immunohistochemical neuroendocrine markers (including cluster of differentiation 56, neural cell adhesion molecules, etc.), and similar oncological outcomes (including metastatic behavior and poor prognosis) [2-6]. However, of note, several recent studies with aid of the next-generation sequencing and cell-free DNA detection found that LCNEC also comprised non-SCLC (NSCLC)-like subset, which may cause the individual response to different chemotherapy regimen; and the common driver mutations, including epidermal growth factor receptor (EGFR) mutation, anaplastic lymphoma kinase (ALK) rearrangements etc., were rarely detected $[4,7]$. Therefore, systemic treatment for pulmonary LCNEC remains under debate in clinical practice $[4,5,7]$. Based on this fact, surgical resection is still recognized as the standard scheme for early-staged pulmonary LCNEC patients to obtain long-term survival [8, 9].

In 1995, a pivotal randomized trial administered by the Lung Cancer Study Group (LCSG) revealed higher localrecurrence rate and inferior OS rate in patients who received sublobar resection (SLR, including non-anatomical 
wedge resection [WR] and anatomical segmentectomy) rather than lobectomy for stage I NSCLC [10]. Since then, lobectomy with mediastinal lymph nodes dissection as the criterial surgical procedure even in stage IA NSCLC has been upheld in the guidelines until recently [8, 9]. However, several retrospective studies have disputed this dogma by demonstrating non-inferior oncological outcomes of SLR and lobectomy for select patientswith stage IA (with or without less than $2 \mathrm{~cm}$ ) NSCLC [11-14]. Also, for stage IA SCLC patients in the Survenillance, Epidemiology, and End Results (SEER) database, there is no statistically difference of cancerspecific survival (CSS) or OS between SLR group and lobectomy group [15]. Potential benefits of SLR include preserving cardiopulmonary function, reducing perioperative morbidity and mortality, and providing chance for repetitive resections $[13,16,17]$. On the other hand, a debate remains about the lower rate of local recurrence after lobectomy that might bring survival benefit, particularly in patients with a good physical status. Specifically, due to the low incidence rate of pulmonary LCNEC and the majority of first diagnosed patients in the advanced stages, the investigations on the oncological clearance of SLR and the equivalency between SLR and lobectomy among pulmonary LCNEC patients remain scarce $[2,18]$. Therefore, in this present study, the data retrieved from the SEER registry was used to compare the oncological outcomes following SLR and lobectomy in stage IA pulmonary LCNEC.

\section{Methods}

\section{Study cohort}

The public-access SEER database with additional treatment fields (1975-2016 varying) used in this study was released on April 2019, which covered approximately $34.6 \%$ of the U.S. population [19]. Patients diagnosed with pulmonary LCNEC (International Classification of Disease for Oncology, 3rd edition [ICD-0-3]: lung and bronchus; and ICD-0-3 histology code: 8013/3) between 1998 and 2016 were extracted by using SEER*Stat (version 8.3.6). Then, we limited all patients with pathological stage IA. In addition, we also excluded patients: (I) with age at diagnosis < 18 years old; (II) with other primary tumor(s); (III) did not receive surgery; (IV) received neoadjuvant or intraoperative radiation; $(\mathrm{V})$ received pneumonectomy or bilobectomy; $(\mathrm{VI})$ with unknown lymph nodes status; (VII) with unknown tumor size; (VIII) with incomplete follow-up data or outcomes. Finally, only 308 patients diagnosed with stage IA LCNEC and underwent WR (surgery of primary site [SPS] codes: 21), segmentectomy (SPS code: 22), or lobectomy (SPS codes: 30 and 33) were retained into the statistical analysis (Fig. 1).

\section{Variable Definitions}

The continuous variable of age at diagnosis was grouped into three groups according to the WHO recommendations on physical activity for health: less than 60 years old, 61-74 years old, and 75 years old and above. Year of diagnosis was divided into 4-year intervals as follows: 2001-2004, 2005-2008, 2009-2012, and 2012-2016. The insurance status of insured, any medicaid, and insured but no specifics were all defined as insured; similarly, the marital status of single included never married, widowed, divorced, and single (never married). In addition, all patients were restaged to IA1, IA2, and IA3 on basis of the American Joint Committee on Cancer 8th Tumor-Nodes-Metastasis (TNM) staging manual for lung cancer. 


\section{Outcomes}

Survival time, measured in months, was calculated from the day of surgical resection to the day of death or the day of last follow-up if patient was documented as alive. Only patients occurred LCNEC-related death were recorded as uncensored in CSS, but OS included any cause of death. The operative mortality was defined as any death within 30 days of surgical resection.

\section{Statistical analysis}

The best cutoff values for tumor size $(\leq 20 \mathrm{~mm} />20, \leq 30 \mathrm{~mm}$ ) and the number of lymph nodes dissection ( $\leq$ $5 / \geq 6$ ) were determined by the X-tile software (version 3.6.1, copyright Yale University 2003). The continuous variables were presented as mean \pm standard deviation (SD) and were compared by the Student's $t$ test between the SLR set and lobectomy set. For categorical variables, the chi-squared test or Fisher's exact test was applied to compare the proportions between the two sets. Survival distributions between variables were analyzed by the Kaplan-Meier method. Thereafter, potential predictors $(P<0.05$, by the log-rank test) identified by univariate analysis for CSS and OS were enrolled into to the Cox proportional hazards regression models to calculate the hazard ratios (HRs).

All above statistical analyses were calculated by using SPSS software (version 24.0, IBM SPSS, Armonk, NY, USA), and the threshold value for significance was less than 0.05 at two sides. With aid of the $\mathrm{R}$ software (version 3.6.1), propensity score matching (PSM) was implemented by the "matchit" package to reduce potential bias between the SLR group and the lobectomy group, and the 1-to-1 nearest neighbor matching (ratio $=1$ ) was set. In addition, the survival curves before and after PSM were plotted by the "survival" and "survminer" packages.

\section{Results}

\section{Patient characteristics}

A total of 308 patients diagnosed with stage IA LCNEC of the lung met inclusion criteria were finally retained in this study (Fig. 1). In demographic characteristics (Tables 1 and 2), the average and median age at diagnosis of whole cohort were all 67 years old (range, 39-89 years old), and most of the patients were white race (88.6\%), female (55.8\%), married (54.2\%), and insured (68.8\%). In addition, the pulmonary LCNEC was mostly located in the right lower lobe (37.0\%), followed by right upper lobe (30.8\%), left lower lobe (15.6\%), right middle lobe $(10.7 \%)$ and left upper lobe $(5.8 \%)$. 
Table 1

Patients' characteristics between sublobar resection and lobectomy group before propensity score matching

\begin{tabular}{|c|c|c|c|}
\hline Variables & $\begin{array}{l}\text { Sublobar resection }(n= \\
79)\end{array}$ & $\begin{array}{l}\text { Lobectomy }(n= \\
229)\end{array}$ & $\begin{array}{l}P \\
\text { value }\end{array}$ \\
\hline Age at diagnosis, (mean \pm SD) & $69.91 \pm 9.47$ & $65.51 \pm 8.64$ & $\begin{array}{l}< \\
0.001\end{array}$ \\
\hline \multicolumn{4}{|l|}{ Year of diagnosis, n (\%) } \\
\hline $2001-2004$ & $15(19.0 \%)$ & $30(13.1 \%)$ & \multirow[t]{4}{*}{0.271} \\
\hline $2005-2008$ & $22(27.8 \%)$ & $61(26.6 \%)$ & \\
\hline $2009-2012$ & $23(29.1 \%)$ & $58(25.3 \%)$ & \\
\hline $2012-2016$ & $19(24.1 \%)$ & $80(34.9 \%)$ & \\
\hline \multicolumn{4}{|l|}{ Race, n (\%) } \\
\hline White & $70(88.6 \%)$ & $203(88.6 \%)$ & \multirow[t]{3}{*}{0.685} \\
\hline Black & $6(7.6 \%)$ & $21(9.2 \%)$ & \\
\hline Asian or Pacific Islander & $3(3.8 \%)$ & $5(2.2 \%)$ & \\
\hline \multicolumn{4}{|l|}{ Sex, n (\%) } \\
\hline Female & $39(49.4 \%)$ & $133(58.1 \%)$ & \multirow[t]{2}{*}{0.179} \\
\hline Male & 40 (50.6\%) & $96(41.9 \%)$ & \\
\hline \multicolumn{4}{|l|}{ Primary site, n (\%) } \\
\hline Upper lobe & $51(64.6 \%)$ & 158 (69.0\%) & \multirow[t]{3}{*}{0.140} \\
\hline Middle lobe & $2(2.5 \%)$ & $16(7.0 \%)$ & \\
\hline Lower lobe & $26(32.9 \%)$ & $55(24.0 \%)$ & \\
\hline \multicolumn{4}{|l|}{ Laterality, n (\%) } \\
\hline Right & $40(50.6 \%)$ & $140(61.1 \%)$ & \multirow[t]{2}{*}{0.102} \\
\hline Left & $39(49.4 \%)$ & $89(38.9 \%)$ & \\
\hline \multicolumn{4}{|l|}{ Grade, n (\%) } \\
\hline Well differentiated, I & $2(2.5 \%)$ & $1(0.4 \%)$ & \multirow[t]{5}{*}{0.465} \\
\hline Moderately differentiated, II & $3(3.8 \%)$ & $6(2.6 \%)$ & \\
\hline Poorly differentiated, III & $37(46.8 \%)$ & $122(53.3 \%)$ & \\
\hline Undifferentiated, IV & $13(16.5 \%)$ & $33(14.4 \%)$ & \\
\hline Unknown & $24(30.4 \%)$ & $67(29.3 \%)$ & \\
\hline
\end{tabular}

SD, standard deviation. 


\begin{tabular}{|c|c|c|c|}
\hline Variables & $\begin{array}{l}\text { Sublobar resection }(n= \\
79)\end{array}$ & $\begin{array}{l}\text { Lobectomy }(n= \\
\text { 229) }\end{array}$ & $\begin{array}{l}P \\
\text { value }\end{array}$ \\
\hline \multicolumn{4}{|l|}{ TNM staging } \\
\hline IA1 & $16(20.3 \%)$ & $36(15.7 \%)$ & \multirow[t]{3}{*}{0.192} \\
\hline IA2 & $43(54.4 \%)$ & $110(48.0 \%)$ & \\
\hline IA3 & $20(25.3 \%)$ & $83(36.2 \%)$ & \\
\hline Tumor size $(\mathrm{mm}$, mean $\pm \mathrm{SD})$ & $16.08 \pm 6.21$ & $18.19 \pm 6.30$ & 0.010 \\
\hline $\begin{array}{l}\text { Number of lymph nodes dissection (mean } \pm \\
\text { SD) }\end{array}$ & $2.91 \pm 4.91$ & $8.91 \pm 8.27$ & $\begin{array}{l}< \\
0.001\end{array}$ \\
\hline \multicolumn{4}{|l|}{ Lymph nodes dissection, $\mathrm{n}(\%)$} \\
\hline Yes & $46(58.2 \%)$ & $211(92.1 \%)$ & \multirow{2}{*}{$\dot{0} 001$} \\
\hline No & $33(41.8 \%)$ & $18(7.9 \%)$ & \\
\hline \multicolumn{4}{|l|}{ Radiation, n (\%) } \\
\hline Radiation after surgery & $3(3.8 \%)$ & $11(4.8 \%)$ & \multirow[t]{2}{*}{0.711} \\
\hline No radiation & $76(96.2 \%)$ & $218(95.2 \%)$ & \\
\hline \multicolumn{4}{|l|}{ Chemotherapy, n (\%) } \\
\hline Yes & $8(10.1 \%)$ & $32(14.0 \%)$ & \multirow[t]{2}{*}{0.380} \\
\hline No/unknown & $71(89.9 \%)$ & $197(86.0 \%)$ & \\
\hline \multicolumn{4}{|l|}{ Insurance, n (\%) } \\
\hline Any insured & $53(67.1 \%)$ & $159(69.4 \%)$ & \multirow[t]{3}{*}{0.416} \\
\hline Uninsured & $0(0.0 \%)$ & $4(1.7 \%)$ & \\
\hline Unknown & $26(32.9 \%)$ & $66(28.8 \%)$ & \\
\hline \multicolumn{4}{|l|}{ Marital status, n (\%) } \\
\hline Single & $32(40.5 \%)$ & $122(53.3 \%)$ & \multirow[t]{3}{*}{0.844} \\
\hline Married & $45(57.0 \%)$ & $100(43.7 \%)$ & \\
\hline Unknown & $2(2.5 \%)$ & $7(3.1 \%)$ & \\
\hline
\end{tabular}


Table 2

Univariate and multivariate analysis of cancer-specific survival and overall survival before propensity score matching

\begin{tabular}{|c|c|c|c|c|c|c|c|c|c|}
\hline \multirow[t]{3}{*}{ Variables } & \multirow[t]{3}{*}{$\mathbf{N}$} & \multicolumn{4}{|c|}{ Univariate analysis } & \multicolumn{4}{|c|}{ Multivariate analysis } \\
\hline & & \multicolumn{2}{|c|}{$\begin{array}{l}\text { Cancer-specific } \\
\text { survival }\end{array}$} & \multicolumn{2}{|c|}{$\begin{array}{l}\text { Overall } \\
\text { survival }\end{array}$} & \multicolumn{2}{|c|}{$\begin{array}{l}\text { Cancer-specific } \\
\text { survival }\end{array}$} & \multicolumn{2}{|c|}{ Overall survival } \\
\hline & & $\begin{array}{l}\text { 5-year } \\
\text { SR (\%) }\end{array}$ & $\begin{array}{l}P \\
\text { value }\end{array}$ & $\begin{array}{l}\text { 5-year } \\
\text { SR } \\
(\%)\end{array}$ & $\begin{array}{l}P \\
\text { value }\end{array}$ & $\begin{array}{l}\mathrm{HR} \\
(95 \% \mathrm{Cl})\end{array}$ & $\begin{array}{l}P \\
\text { value }\end{array}$ & $\begin{array}{l}\text { HR } \\
(95 \% \mathrm{Cl})\end{array}$ & $\begin{array}{l}P \\
\text { value }\end{array}$ \\
\hline \multicolumn{10}{|l|}{$\begin{array}{l}\text { Age at } \\
\text { diagnosis }\end{array}$} \\
\hline$<60$ years old & 79 & $70.3 \%$ & \multirow[t]{2}{*}{0.014} & $64.8 \%$ & \multirow{2}{*}{$\begin{array}{l}< \\
0.001\end{array}$} & Reference & & \multicolumn{2}{|l|}{ Reference } \\
\hline $60-74$ years old & 161 & $68.5 \%$ & & $54.4 \%$ & & $\begin{array}{l}1.210 \\
(1.013- \\
1.556)\end{array}$ & 0.043 & $\begin{array}{l}1.623 \\
(1.043- \\
2.526)\end{array}$ & 0.032 \\
\hline$\geq 75$ years old & 68 & $49.8 \%$ & & $33.0 \%$ & & $\begin{array}{l}2.012 \\
(1.109- \\
3.650)\end{array}$ & 0.021 & $\begin{array}{l}2.588 \\
(1.560- \\
4.291)\end{array}$ & $\begin{array}{l}< \\
0.001\end{array}$ \\
\hline
\end{tabular}

\section{Year of}

diagnosis

\begin{tabular}{|c|c|c|c|c|}
\hline $2001-2004$ & 45 & $62.1 \%$ & 0.287 & $42.2 \%$ \\
\hline $2005-2008$ & 83 & $63.7 \%$ & & $53.0 \%$ \\
\hline $2009-2012$ & 81 & $62.6 \%$ & & $51.9 \%$ \\
\hline $2012-2016$ & 99 & NR & & NR \\
\hline
\end{tabular}

\section{Race}

\begin{tabular}{|c|c|c|c|c|}
\hline White & 273 & $65.3 \%$ & 0.732 & $53.3 \%$ \\
\hline Black & 27 & $53.8 \%$ & & $41.4 \%$ \\
\hline $\begin{array}{l}\text { Asian or Pacific } \\
\text { Islander }\end{array}$ & 8 & $85.7 \%$ & & $57.1 \%$ \\
\hline
\end{tabular}

\section{Sex}

\begin{tabular}{|c|c|c|c|c|}
\hline Female & 172 & $69.3 \%$ & 0.267 & $56.1 \%$ \\
\hline Male & 136 & $59.2 \%$ & & $47.5 \%$ \\
\hline
\end{tabular}

Primary site

\begin{tabular}{|c|c|c|c|c|}
\hline Upper lobe & 209 & $69.4 \%$ & 0.505 & $54.1 \%$ \\
\hline Middle lobe & 18 & $51.3 \%$ & & $51.3 \%$ \\
\hline Lower lobe & 81 & $56.3 \%$ & & $47.6 \%$ \\
\hline
\end{tabular}

SR, survival rate; No., number; $\mathrm{HR}$, hazard ratio; $\mathrm{Cl}$, confidence interval; $\mathrm{NR}$, no reached; 


\begin{tabular}{|c|c|c|c|c|c|c|c|c|c|}
\hline \multirow[t]{3}{*}{ Variables } & \multirow[t]{3}{*}{$\mathbf{N}$} & \multicolumn{4}{|c|}{ Univariate analysis } & \multicolumn{4}{|c|}{ Multivariate analysis } \\
\hline & & \multicolumn{2}{|c|}{$\begin{array}{l}\text { Cancer-specific } \\
\text { survival }\end{array}$} & \multicolumn{2}{|c|}{$\begin{array}{l}\text { Overall } \\
\text { survival }\end{array}$} & \multicolumn{2}{|c|}{$\begin{array}{l}\text { Cancer-specific } \\
\text { survival }\end{array}$} & \multicolumn{2}{|c|}{ Overall survival } \\
\hline & & $\begin{array}{l}\text { 5-year } \\
\text { SR (\%) }\end{array}$ & $\begin{array}{l}\mathrm{P} \\
\text { value }\end{array}$ & $\begin{array}{l}\text { 5-year } \\
\text { SR } \\
(\%)\end{array}$ & $\begin{array}{l}\mathrm{P} \\
\text { value }\end{array}$ & $\begin{array}{l}\mathrm{HR} \\
(95 \% \mathrm{Cl})\end{array}$ & $\begin{array}{l}\mathrm{P} \\
\text { value }\end{array}$ & $\begin{array}{l}\mathrm{HR} \\
(95 \% \mathrm{Cl})\end{array}$ & $\begin{array}{l}P \\
\text { value }\end{array}$ \\
\hline \multicolumn{10}{|l|}{ Laterality } \\
\hline Right & 180 & $58.0 \%$ & \multirow[t]{2}{*}{0.003} & $47.6 \%$ & \multirow[t]{2}{*}{0.046} & Reference & & \multicolumn{2}{|c|}{ Reference } \\
\hline Left & 128 & $74.4 \%$ & & $58.8 \%$ & & $\begin{array}{l}0.512 \\
(0.330- \\
0.795)\end{array}$ & 0.003 & $\begin{array}{l}0.704 \\
(0.508- \\
0.975)\end{array}$ & 0.035 \\
\hline \multicolumn{10}{|l|}{ Grade } \\
\hline $\begin{array}{l}\text { Well } \\
\text { differentiated, I }\end{array}$ & 3 & $100.0 \%$ & \multirow[t]{5}{*}{0.686} & $0.0 \%$ & \multirow[t]{5}{*}{0.937} & & & & \\
\hline $\begin{array}{l}\text { Moderately } \\
\text { differentiated, II }\end{array}$ & 9 & $60.0 \%$ & & $53.3 \%$ & & & & & \\
\hline $\begin{array}{l}\text { Poorly } \\
\text { differentiated, III }\end{array}$ & 159 & $64.3 \%$ & & $53.3 \%$ & & & & & \\
\hline $\begin{array}{l}\text { Undifferentiated, } \\
\text { IV }\end{array}$ & 46 & $63.0 \%$ & & $46.4 \%$ & & & & & \\
\hline Unknown & 91 & $67.1 \%$ & & $52.2 \%$ & & & & & \\
\hline \multicolumn{10}{|l|}{ TNM staging } \\
\hline IA1 & 52 & $61.4 \%$ & \multirow[t]{3}{*}{0.509} & $52.3 \%$ & \multirow[t]{3}{*}{0.160} & & & & \\
\hline IA2 & 153 & $66.6 \%$ & & $54.9 \%$ & & & & & \\
\hline IA3 & 103 & $63.7 \%$ & & $48.2 \%$ & & & & & \\
\hline \multicolumn{10}{|l|}{ Tumor size } \\
\hline$\leq 20 \mathrm{~mm}$ & 205 & $65.7 \%$ & \multirow[t]{2}{*}{0.552} & $54.5 \%$ & \multirow[t]{2}{*}{0.102} & & & & \\
\hline$\nabla 20, \leq 30 \mathrm{~mm}$ & 103 & $63.7 \%$ & & $48.2 \%$ & & & & & \\
\hline
\end{tabular}

SR, survival rate; No., number; $\mathrm{HR}$, hazard ratio; $\mathrm{Cl}$, confidence interval; $\mathrm{NR}$, no reached; 


\begin{tabular}{|c|c|c|c|c|c|c|c|c|c|}
\hline \multirow[t]{3}{*}{ Variables } & \multirow[t]{3}{*}{$\mathbf{N}$} & \multicolumn{4}{|c|}{ Univariate analysis } & \multicolumn{4}{|c|}{ Multivariate analysis } \\
\hline & & \multicolumn{2}{|c|}{$\begin{array}{l}\text { Cancer-specific } \\
\text { survival }\end{array}$} & \multicolumn{2}{|c|}{$\begin{array}{l}\text { Overall } \\
\text { survival }\end{array}$} & \multicolumn{2}{|c|}{$\begin{array}{l}\text { Cancer-specific } \\
\text { survival }\end{array}$} & \multicolumn{2}{|c|}{ Overall survival } \\
\hline & & $\begin{array}{l}\text { 5-year } \\
\text { SR (\%) }\end{array}$ & $\begin{array}{l}P \\
\text { value }\end{array}$ & $\begin{array}{l}\text { 5-year } \\
\text { SR } \\
(\%)\end{array}$ & $\begin{array}{l}P \\
\text { value }\end{array}$ & $\begin{array}{l}\mathrm{HR} \\
(95 \% \mathrm{Cl})\end{array}$ & $\begin{array}{l}P \\
\text { value }\end{array}$ & $\begin{array}{l}\mathrm{HR} \\
(95 \% \mathrm{Cl})\end{array}$ & $\begin{array}{l}P \\
\text { value }\end{array}$ \\
\hline \multicolumn{10}{|l|}{$\begin{array}{l}\text { Lymph nodes } \\
\text { dissection }\end{array}$} \\
\hline Yes & 257 & $66.5 \%$ & \multirow[t]{2}{*}{0.093} & $52.9 \%$ & \multirow[t]{2}{*}{0.120} & & & & \\
\hline No & 51 & $57.3 \%$ & & $49.0 \%$ & & & & & \\
\hline \multicolumn{10}{|l|}{$\begin{array}{l}\text { No. of lymph } \\
\text { nodes } \\
\text { dissection }\end{array}$} \\
\hline$\leq 5$ & 156 & $58.5 \%$ & \multirow[t]{2}{*}{0.003} & $46.6 \%$ & \multirow[t]{2}{*}{0.013} & Reference & & \multicolumn{2}{|l|}{ Reference } \\
\hline$\geq 6$ & 152 & $72.8 \%$ & & $59.5 \%$ & & $\begin{array}{l}0.622 \\
(0.385- \\
0.994)\end{array}$ & 0.042 & $\begin{array}{l}0.774 \\
(0.539- \\
1.112)\end{array}$ & 0.167 \\
\hline \multicolumn{10}{|l|}{ Radiation } \\
\hline $\begin{array}{l}\text { Radiation after } \\
\text { surgery }\end{array}$ & 14 & $58.0 \%$ & \multirow[t]{2}{*}{0.057} & $52.2 \%$ & \multirow[t]{2}{*}{0.506} & Reference & & & \\
\hline No radiation & 294 & $65.3 \%$ & & $52.3 \%$ & & $\begin{array}{l}0.585 \\
(0.289- \\
1.183)\end{array}$ & 0.135 & & \\
\hline \multicolumn{10}{|l|}{ Chemotherapy } \\
\hline Yes & 40 & $78.3 \%$ & \multirow[t]{2}{*}{0.376} & $72.7 \%$ & \multirow[t]{2}{*}{0.078} & & & & \\
\hline No/unknown & 268 & $63.0 \%$ & & $49.6 \%$ & & & & & \\
\hline \multicolumn{10}{|l|}{ Insurance } \\
\hline Any insured & 212 & $65.4 \%$ & \multirow[t]{3}{*}{0.506} & $54.2 \%$ & \multirow[t]{3}{*}{0.479} & & & & \\
\hline Uninsured & 4 & $50.0 \%$ & & $50.0 \%$ & & & & & \\
\hline Unknown & 92 & $64.3 \%$ & & $47.5 \%$ & & & & & \\
\hline \multicolumn{10}{|l|}{ Marital status } \\
\hline Single & 132 & $70.2 \%$ & \multirow[t]{3}{*}{0.123} & $55.5 \%$ & \multirow[t]{3}{*}{0.487} & & & & \\
\hline Married & 167 & $59.9 \%$ & & $49.2 \%$ & & & & & \\
\hline Unknown & 9 & $100.0 \%$ & & $80.0 \%$ & & & & & \\
\hline
\end{tabular}

SR, survival rate; No., number; $\mathrm{HR}$, hazard ratio; $\mathrm{Cl}$, confidence interval; $\mathrm{NR}$, no reached; 


\begin{tabular}{|c|c|c|c|c|c|c|c|c|c|}
\hline \multirow[t]{3}{*}{ Variables } & \multirow[t]{3}{*}{$\mathbf{N}$} & \multicolumn{4}{|c|}{ Univariate analysis } & \multicolumn{4}{|c|}{ Multivariate analysis } \\
\hline & & \multicolumn{2}{|c|}{$\begin{array}{l}\text { Cancer-specific } \\
\text { survival }\end{array}$} & \multicolumn{2}{|c|}{$\begin{array}{l}\text { Overall } \\
\text { survival }\end{array}$} & \multicolumn{2}{|c|}{$\begin{array}{l}\text { Cancer-specific } \\
\text { survival }\end{array}$} & \multicolumn{2}{|c|}{ Overall survival } \\
\hline & & $\begin{array}{l}\text { 5-year } \\
\text { SR (\%) }\end{array}$ & $\begin{array}{l}P \\
\text { value }\end{array}$ & $\begin{array}{l}\text { 5-year } \\
\text { SR } \\
(\%)\end{array}$ & $\begin{array}{l}P \\
\text { value }\end{array}$ & $\begin{array}{l}\mathrm{HR} \\
(95 \% \mathrm{Cl})\end{array}$ & $\begin{array}{l}P \\
\text { value }\end{array}$ & $\begin{array}{l}\mathrm{HR} \\
(95 \% \mathrm{Cl})\end{array}$ & $\begin{array}{l}P \\
\text { value }\end{array}$ \\
\hline $\begin{array}{l}\text { Sublobar } \\
\text { resection }\end{array}$ & 79 & $56.5 \%$ & 0.037 & $42.9 \%$ & 0.019 & Reference & & Reference & \\
\hline Lobectomy & 229 & $67.8 \%$ & & $55.7 \%$ & & $\begin{array}{l}0.585 \\
(0.289- \\
1.183)\end{array}$ & 0.135 & $\begin{array}{l}0.815 \\
(0.560- \\
1.186)\end{array}$ & 0.285 \\
\hline
\end{tabular}

In surgical operation (Tables 1 and 2), 229 patients underwent lobectomy, 62 patients underwent WR, and only 17 patients underwent segementectomy. $83.4 \%$ of the patients $(257 / 308)$ received at least one lymph node dissection (mean, 7.37; range, 0-55). In pathological examination, the mean tumor size was 17.65 millimeter ( $\mathrm{mm}$; range, 7-30 mm), and the patients diagnosed with stage $\mathrm{T} 1 \mathrm{a}, \mathrm{T} 1 \mathrm{~b}$ and $\mathrm{T} 1 \mathrm{c}$ were 52 cases, 153 cases, and 103 cases, respectively. Poorly differentiated and undifferentiated LCNEC observed under microscope accounted for the majority ( $51.6 \%$ and $14.9 \%$, respectively). In systemic treatment, 14 patients (including 3 WRs and 11 lobectomies) received postoperative radiation and 40 patients (including 8 WRs and 32 lobectomies) received chemotherapy.

\section{Slr Versus Lobectomy}

Patients who underwent lobectomy were younger ( $65.51 \pm 8.64$ vs. $69.91 \pm 9.47, \mathrm{P}<0.001)$, but showed larger tumor size $(18.19 \pm 6.30$ vs. $16.08 \pm 6.21, P=0.010)$ than those who underwent SLR. In addition, the percentage of patients receiving lymph nodes dissection in the SLR group was significant lower than that in the lobectomy group ( $58.2 \%$ vs. $92.1 \%, \mathrm{P}<0.01)$; similarly, SLR patients had a less number of lymph nodes dissected $(2.91 \pm$ 4.91 vs. $8.91 \pm 827, \mathrm{P}<0.001)$. The differences between the two groups are further detailed in Table 1 . In addition, there were three patients occurred death within 30 days after lobectomy and only one patient occurred operative mortality after SLR $(P=0.976)$.

On Kaplan-Meier survival analysis, patients who underwent SLR showed worse 5-year CSS rate and OS rate ( $56.5 \%$ vs. $67.8 \%, P=0.037 ; 42.9 \%$ vs. $55.7 \%, P=0.019$; Table 2 ). Separately, CSS in patients with WR or segmentectomy was worse than those with lobectomy in trend (WR: $60.4 \%$ vs. $67.8 \%, P=0.084$; segmentectomy: $40.0 \%$ vs. $67.8 \%, P=0.095$; Fig. $2 a$ ). However, WR and segmentectomy were significantly associated with worse 5-year OS rate compared to lobectomy (WR: $46.9 \%$ vs. $55.7 \%, P=0.023$; segmentectomy: $26.5 \%$ vs. $55.7 \%, P=0.083$; Fig. $2 b$ ). Unexpectedly, after Cox multivariate regression analysis, there were no significant difference between the SLR group and the lobectomy group in CSS or OS (all P >0.05, Table 2). 
The imbalanced variables, including age at diagnosis, tumor size and lymph nodes dissection (yes/no), were included into the 1-to-1 PSM. After that, a total of 158 patients were selected, with 79 patients in both the SLR group and lobectomy group. No significant difference was observed between the two groups in 13 of the 15 variables after PSM, except for whether to dissect lymph nodes and the number of lymph nodes dissection (Table S1). On Kaplan-Meier survival analysis, there was no significant difference of CSS or OS between the two matched groups as well (all $P>0.05$, Fig. $2 c$ and $2 d$ ).

\section{Prognostic Factors For Css And Os}

The median follow-up time of the 308 patients was 33.5 months (range from 1 month to 191 months). The 3year and 5-year CSS and OS rates were 73.5\% and 63.6\%, 64.9\% and 52.4\%, respectively. Table 2 showed the univariate and multivariate analyses. Age at diagnosis (Fig. 3a and 3b) and laterality of tumor (Fig. 3c and 3d) were identified as independent prognostic factors for CSS and OS. In addition, the number of lymph nodes dissection less than 5 was a risk factor for CSS (Fig. 3e), but not for OS.

\section{Discussion}

The present study was conducted to investigate the CSS and OS associated with SLR and lobectomy in 308 patients with stage IA LCNEC retrieved from the National Cancer Institute's SEER database, by using Cox proportional hazards regression model and PSM to adjust the potential confounding factors. We found that SLR was not inferior to lobectomy for CSS and OS. However, multivariate analyses demonstrated that advancing age and right-sided lung resection were significant risk factors for CSS and OS; in addition, patients receiving lymph nodes dissection less than five was associated with decreased CSS.

In line with the published literatures on patients with stage IA LECNEC of the lung who underwent lobectomy (27.0-67.0\%), the 5-year OS rate was $67.8 \%$ in this population-based study, which was worse than other NSCLC $[18,20-23]$. However, owing to the low incidence and a lack of evidence-based surgical treatment for LCNEC, there was limited study to evaluate the survival after SLR $[6,18,22,24]$. Reported by the NETs Working Group, 24 patients collected from eight high-volume European Thoracic Surgery Institutions received SLR, including 13 WR and 11 lobectomy; furthermore, compared to 81 patients received lobectomy, these patients received SLR showed a equivalent 5-year OS rate [6]. Similar survival outcome was vertified by another single-institution study, which was the largest study cohort between 1991 and 2006 [22]. Conversely, in 2019, a prognostic analysis comparing SLR (133 cases) versus lobectomy or bilobectomy (425 cases) utilizing the SEER database found the five-year OS rate was $22.5 \%$ and $42.5 \%$, respectively $(P<0.001)$ [24]. However, of note, patients diagnosed with stage I through IV were all enrolled in above studies and most of the statistical analyses were performed on small size samples.

Recently, Waseem et al. identified 1011 patients diagnosed with stage I LCNEC ( $\leq 3 \mathrm{~cm})$ from the National Cancer Database (NCDB) between 2004 and 2014, and the findings showed patients who underwent lobectomy had better OS when compared with those with SLR (before PSM: $56.6 \%$ vs. $37.9 \%, P<0.001$; after PSM: $60.3 \%$ vs. $41.5 \%, P=0.001$ ), which was the largest study cohort for stage I LCNEC until now [18]. The constituent ratio of WR, segmentectomy and lobectomy for stage IA LCNEC was parallel between the NCDB database and the SEER database $(22.1 \%, 4.0 \%$ and $74.0 \%$ vs. $20.1 \%, 5.5 \%$ and $74.4 \%$, respectively). In addition, 
fully consistent with our findings, patients receiving SLR were more likely to have increased age, smaller size, and less likely to have lymph nodes dissection than those receiving lobectomy. However, SLR showed no inferior to lobectomy for CSS and OS of stage IA LCNEC by multivariate analysis and propensity score analysis in present study. We speculate that only PSM performed in the study of Waseem et al. may strengthen the power to identify statistical difference in survival. As known, if the ratio of the patients in control group (lobectomy) to the patients in study group (SLR) is less than 10-to-1, plenty of patients in study group could not match the nearest control cases during the process of PSM, which may increase the man-made selection bias [25]. Therefore, it is necessary to conduct the multivariate analysis to adjust potential confounding factors before PSM and/or after PSM $[2,13,23,25]$. Additionally, the reasons for SLR include intention-to-treat and compromise-to-treat in clinical practice, and the intentional SLR is appropriate for patients with poor pulmonary reserve or other major comorbidity; moreover, the sufficient parenchymal resection margin is vital when surgeons perform the SLR [26]. Regrettably, these variables were not available within the SEER database. On the whole, the above weaknesses in the published and our studies may be preliminarily explain why the results were different between the two large size samples, and the debate on the oncological outcomes following SLR compared to lobectomy for patients with stage IA LCNEC remains [6, 18, 22, 24].

We observed notable difference in the number of lymph nodes dissected by lobectomy and SLR, and the proportion of the patients without lymph nodes dissection in SLR group was significantly higher than that in lobectomy group, which were fully consistent with other studies for comparing lobectomy and SLR for stage IA NSCLC based on the NCDB $[14,27]$. It meant that patients receiving SLR for stage IA lung cancer in the NCDB and SEER database may not receive adequate lymph nodes dissection. Previous studies have found that adequate lymph nodes examination for lung cancer, especially in SLR, was associated with more accurate pathological staging and better survival $[14,27,28]$. Similarly, our result showed that the number of lymph nodes dissection greater than six yielded improved long-term benefit in CSS for stage IA LCNEC patients, and more lymph nodes dissection did not increase the operative mortality. In addition, line with other studies on early-stage LCNEC, Cox regression analysis revealed advancing age to be associated with worse survival [23].

Right-side radiation or pneumonectomy for lung cancer was regarded as independent risk factor for long-term survival $[29,30]$. The study on the right ventricular (RV) response to lung resection by using cardiovascular magnetic resonance imaging, reported by Philip et al., found that RV dysfunction occurred immediately following lung resection, especially right-sided resection, and persisted two months or more, which may associated with the dyspnea and reduced functional capacity [29]. In addition, Carolyn et al. reported that RV end-diastolic volume and center venous pressure would significantly increased after right-sided lung resection as well [30]. Therefore, the right-sided lung resection for early-stage LCNEC may also have negative impact on the cardiopulmonary function, postoperative complications, and long-term survival, which was similar to a prognostic analysis in patients with stage IA SCLC [15].

Undeniably, this population-based study had several limitations. First, the prospective study was difficult to carry out due to the rarity of early-stage LCNEC, therefore, this study was conducted from a retrospective viewpoint. However, multivariate analysis and PSM were performed to reduce the confounding factors and selection bias. Second, several important variables associated with the oncological outcomes after limited resection, such as reason for SLR, preoperative cardiopulmonary function, status of resection margin, local 
recurrence etc, were not documented in the SEER database. Third, limited by the number of patients who underwent SLR for stage IA LCNEC, we could not further compare the outcomes of segmentectomy and WR.

\section{Conclusion}

Although SLR is not inferior to lobectomy in terms of survival for patients with stage IA LCNEC of the lung, more lymph nodes can be dissected during lobectomy. Lobectomy should be condidered as a standard procedure for patients with early-stage LCNEC of the lung who are able to withstand lobectomy.

\section{Abbreviations}

LCNEC: large cell neuroendocrine carcinoma; OS: overall survival; CSS: cancer-specific survival; SLR: sublobar resection; WR: wedge resection; WHO: World Health Organization; NET: neuroendocrine tumor; SCLC: small cell lung cancer; NSCLC: non-small cell lung cancer; DNA: deoxyribonucleic acid; EGFR: epidermal growth factor receptor; ALK: anaplastic lymphoma kinase; SEER: the Survenillance, Epidemiology, and End Results; ICD-0-3: International Classification of Disease for Oncology, 3rd edition; PSM: propensity score matching.

\section{Declarations}

\section{Authors' contributions}

$X Y, Z H, M Z$ and $L Z$ conceived and designed the present study. MZ, YL, RZ, YW, XZ and LY analyzed the data, $\mathrm{RZ}, \mathrm{YW}, \mathrm{XZ}, \mathrm{LY}$ and $\mathrm{DZ}$ interpreted the data and wrote the manuscript. All authors read and approved the final manuscript.

\section{Funding}

This study was found by the National Key Research and Development Plan (No. 2016YFC0905400), the Ministry of Science and Technology of the People's Republic of China.

\section{Availability of data and materials}

Data files were downloaded directly from the SEER website.

\section{Ethics approval and consent to participate}

This retrospective study based on the SEER database was approved by the Surveillance Research Program in National Cancer Institute's Division of Cancer Control and Population Science (DCCPS) (reference No. 12101Nov2018) and the board-certified Research Ethics Committee at Sun Yat-sen University Cancer Center (No. B2018-011).

\section{Consent for publication}

Not applicable.

\section{Competing interests}


The authors have no competing interests to declare.

\section{Author details}

${ }^{1}$ State Key Laboratory of Oncology in South China, Collaborative Innovation Center for Cancer Medicine; Department of Thoracic Surgery, Sun Yat-sen University Cancer Center, Guangzhou 510060, China.

${ }^{2}$ Department of Pathology, Shenzhen Maternity and Child Healthcare Hospital, Shenzhen 518028, China.

${ }^{3}$ Department of Anesthesiology, Sun Yat-sen University Cancer Center, Guangzhou 510060, China.

\section{References}

1. Travis WD, Linnoila RI, Tsokos MG, Hitchcock CL, Cutler GB Jr, Nieman L, Chrousos G, Pass H, Doppman J. Neuroendocrine tumors of the lung with proposed criteria for large-cell neuroendocrine carcinoma. An ultrastructural, immunohistochemical, and flow cytometric study of 35 cases. Am J Surg Pathol. 1991;15(6):529-53.

2. Yang Q, Xu Z, Chen X, Zheng L, Yu Y, Zhao X, Chen M, Luo B, Wang J, Sun J. Clinicopathological characteristics and prognostic factors of pulmonary large cell neuroendocrine carcinoma: A large population-based analysis. Thoracic cancer. 2019;10(4):751-60.

3. Travis WD, Brambilla E, Burke AP, Marx A, Nicholson AG. Introduction to The 2015 World Health Organization Classification of Tumors of the Lung, Pleura, Thymus, and Heart. Journal of thoracic oncology: official publication of the International Association for the Study of Lung Cancer. 2015;10(9):1240-2.

4. Zhuo M, Guan Y, Yang X, Hong L, Wang Y, Li Z, Chen R, Abbas HA, Chang L, Gong Y, et al. The Prognostic and Therapeutic Role of Genomic Subtyping by Sequencing Tumor or Cell-Free DNA in Pulmonary LargeCell Neuroendocrine Carcinoma. Clinical cancer research: an official journal of the American Association for Cancer Research. 2020;26(4):892-901.

5. Zhang JT, Li Y, Yan LX, Zhu ZF, Dong XR, Chu Q, Wu L, Zhang HM, Xu CW, Lin G, et al. Disparity in clinical outcomes between pure and combined pulmonary large-cell neuroendocrine carcinoma: A multi-center retrospective study. Lung cancer (Amsterdam Netherlands). 2020;139:118-23.

6. Filosso PL, Rena O, Guerrera F, Moreno Casado P, Sagan D, Raveglia F, Brunelli A, Welter S, Gust L, Pompili $C$, et al. Clinical management of atypical carcinoid and large-cell neuroendocrine carcinoma: a multicentre study on behalf of the European Association of Thoracic Surgeons (ESTS) Neuroendocrine Tumours of the Lung Working Groupt. European journal of cardio-thoracic surgery: official journal of the European Association for Cardio-thoracic Surgery. 2015;48(1):55-64.

7. Rekhtman N, Pietanza MC, Hellmann MD, Naidoo J, Arora A, Won H, Halpenny DF, Wang H, Tian SK, Litvak AM, et al. Next-Generation Sequencing of Pulmonary Large Cell Neuroendocrine Carcinoma Reveals Small Cell Carcinoma-like and Non-Small Cell Carcinoma-like Subsets. Clinical cancer research: an official journal of the American Association for Cancer Research. 2016;22(14):3618-29.

8. Howington JA, Blum MG, Chang AC, Balekian AA, Murthy SC. Treatment of stage I and II non-small cell lung cancer: Diagnosis and management of lung cancer, 3rd ed: American College of Chest Physicians evidence-based clinical practice guidelines. Chest. 2013;143(5 Suppl):e278S-313S. 
9. Ettinger DS, Wood DE, Aggarwal C, Aisner DL, Akerley W, Bauman JR, Bharat A, Bruno DS, Chang JY, Chirieac LR, et al: NCCN Guidelines Insights: Non-Small Cell Lung Cancer, Version 1.2020. Journal of the National Comprehensive Cancer Network: JNCCN2019, 17(12):1464-1472.

10. Ginsberg RJ, Rubinstein LV. Randomized trial of lobectomy versus limited resection for T1 N0 non-small cell lung cancer. Lung Cancer Study Group. Ann Thorac Surg. 1995;60(3):615-22. discussion 622 - 613.

11. Sienel W, Dango S, Kirschbaum A, Cucuruz B, Hörth W, Stremmel C, Passlick B. Sublobar resections in stage IA non-small cell lung cancer: segmentectomies result in significantly better cancer-related survival than wedge resections. European journal of cardio-thoracic surgery: official journal of the European Association for Cardio-thoracic Surgery. 2008;33(4):728-34.

12. Koike T, Koike T, Yoshiya K, Tsuchida M, Toyabe S. Risk factor analysis of locoregional recurrence after sublobar resection in patients with clinical stage IA non-small cell lung cancer. J Thorac Cardiovasc Surg. 2013;146(2):372-8.

13. Altorki NK, Yip R, Hanaoka T, Bauer T, Aye R, Kohman L, Sheppard B, Thurer R, Andaz S, Smith M, et al. Sublobar resection is equivalent to lobectomy for clinical stage $1 \mathrm{~A}$ lung cancer in solid nodules. $\mathrm{J}$ Thorac Cardiovasc Surg. 2014;147(2):754-62. Discussion 762 - 754.

14. Subramanian M, McMurry T, Meyers BF, Puri V, Kozower BD. Long-Term Results for Clinical Stage IA Lung Cancer: Comparing Lobectomy and Sublobar Resection. Ann Thorac Surg. 2018;106(2):375-81.

15. Gu C, Huang Z, Dai C, Wang Y, Ren Y, She Y, Su H, Chen C. Prognostic Analysis of Limited Resection Versus Lobectomy in Stage IA Small Cell Lung Cancer Patients Based on the Surveillance, Epidemiology, and End Results Registry Database. Frontiers in genetics. 2018;9:568.

16. Zhang R, Wang G, Lin Y, Wen Y, Huang Z, Zhang X, Yu X, Wang W, Xi K, Cerfolio RJ, et al. Extent of resection and lymph node evaluation in early stage metachronous second primary lung cancer: a population-based study. Translational lung cancer research. 2020;9(1):33-44.

17. Villamizar N, Swanson SJ. Lobectomy vs. segmentectomy for NSCLC $(T<2 \mathrm{~cm})$. Annals of cardiothoracic surgery. 2014;3(2):160-6.

18. Lutfi W, Schuchert MJ, Dhupar R, Sarkaria I, Christie NA, Yang CJ, Deng JZ, Luketich JD, Okusanya OT. Sublobar resection is associated with decreased survival for patients with early stage large-cell neuroendocrine carcinoma of the lung. Interact Cardiovasc Thorac Surg. 2019;29(4):517-24.

19. Siegel RL, Miller KD, Jemal A. Cancer statistics, 2020. Cancer J Clin. 2020;70(1):7-30.

20. Iyoda A, Hiroshima K, Moriya Y, Sekine Y, Shibuya K, lizasa T, Nakatani Y, Fujisawa T. Prognostic impact of large cell neuroendocrine histology in patients with pathologic stage la pulmonary non-small cell carcinoma. J Thorac Cardiovasc Surg. 2006;132(2):312-5.

21. lyoda A, Jiang SX, Travis WD, Kurouzu N, Ogawa F, Amano H, Sato Y, Rusch VW, Saegusa M, Satoh Y. Clinicopathological features and the impact of the new TNM classification of malignant tumors in patients with pulmonary large cell neuroendocrine carcinoma. Molecular clinical oncology. 2013;1(3):43743.

22. Veronesi G, Morandi U, Alloisio M, Terzi A, Cardillo G, Filosso P, Rea F, Facciolo F, Pelosi G, Gandini S, et al. Large cell neuroendocrine carcinoma of the lung: a retrospective analysis of 144 surgical cases. Lung cancer (Amsterdam Netherlands). 2006;53(1):111-5. 
23. Kujtan L, Muthukumar V, Kennedy KF, Davis JR, Masood A, Subramanian J. The Role of Systemic Therapy in the Management of Stage I Large Cell Neuroendocrine Carcinoma of the Lung. Journal of thoracic oncology: official publication of the International Association for the Study of Lung Cancer. 2018;13(5):707-14.

24. Cao L, Li ZW, Wang M, Zhang TT, Bao B, Liu YP. Clinicopathological characteristics, treatment and survival of pulmonary large cell neuroendocrine carcinoma: a SEER population-based study. PeerJ. 2019;7:e6539.

25. Austin PC. An Introduction to Propensity Score Methods for Reducing the Effects of Confounding in Observational Studies. Multivar Behav Res. 2011;46(3):399-424.

26. Harrison $S$, Stiles B, Altorki $N$. What is the role of wedge resection for T1a lung cancer? Journal of thoracic disease. 2018;10(Suppl 10):1157-s1162.

27. Khullar OV, Liu Y, Gillespie T, Higgins KA, Ramalingam S, Lipscomb J, Fernandez FG. Survival After Sublobar Resection versus Lobectomy for Clinical Stage IA Lung Cancer: An Analysis from the National Cancer Data Base. Journal of thoracic oncology: official publication of the International Association for the Study of Lung Cancer. 2015;10(11):1625-33.

28. Wu YC, Lin CF, Hsu WH, Huang BS, Huang MH, Wang LS. Long-term results of pathological stage I nonsmall cell lung cancer: validation of using the number of totally removed lymph nodes as a staging control. European journal of cardio-thoracic surgery: official journal of the European Association for Cardio-thoracic Surgery. 2003;24(6):994-1001.

29. McCall PJ, Arthur A, Glass A, Corcoran DS, Kirk A, Macfie A, Payne J, Johnson M, Kinsella J, Shelley BG. The right ventricular response to lung resection. J Thorac Cardiovasc Surg. 2019;158(2):556-65.e555.

30. Reed CE, Dorman BH, Spinale FG. Assessment of right ventricular contractile performance after pulmonary resection. Ann Thorac Surg. 1993;56(3):426-31. discussion 431 - 422.

\section{Figures}




\section{Surveillance, Epidemiology, and End Results (SEER) 18 Registries with additional} treatment fields (1975-2016 varying), released April 2019.

ICD-O-3 Site record: lung and bronchus

-ICD-O-3 Histology/Behavior: 8013/3 large cell neuroendocrine carcinoma (LCNEC).

$$
(\mathrm{N}=4698)
$$

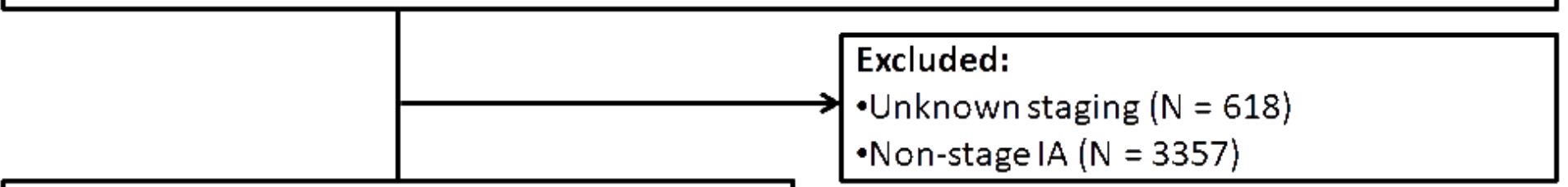

\section{Patients with Stage IA LCNEC (N = 723)}

\section{Patients with Stage IA LCNEC ( $=319)$}

Excluded:

- With other primary cancer $(\mathrm{s})(\mathrm{N}=252)$

-Surgery not performed $(\mathrm{N}=98)$

-Pneumonectomy or bilobectomy $(\mathrm{N}=52)$

-With induction therapy $(\mathrm{N}=2)$

- Restaging according to the American Joint Committee on Cancer 8th TNM staging manual for lung cancer.

\section{Excluded:}

- Unknown tumor size $(\mathrm{N}=11)$

\begin{tabular}{|c|}
\hline $\begin{array}{c}\text { Patients with Stage IA1-3 LCNEC }(\mathrm{N}=308) \\
\text { Wedge resection }(\mathrm{N}=62) \\
\text { Segemntectomy }(\mathrm{N}=17) \\
\text { Lobectomy }(\mathrm{N}=229)\end{array}$ \\
\hline \\
\hline $\begin{array}{c}\text { After matching }(\mathrm{N}=158) \\
\text { Wedge resection }(\mathrm{N}=62) \\
\text { Segemntectomy }(N=17) \\
\text { Lobectomy }(\mathrm{N}=79)\end{array}$ \\
\hline
\end{tabular}

Figure 1

The flow diagram of the selection process for this population-based study cohort. 

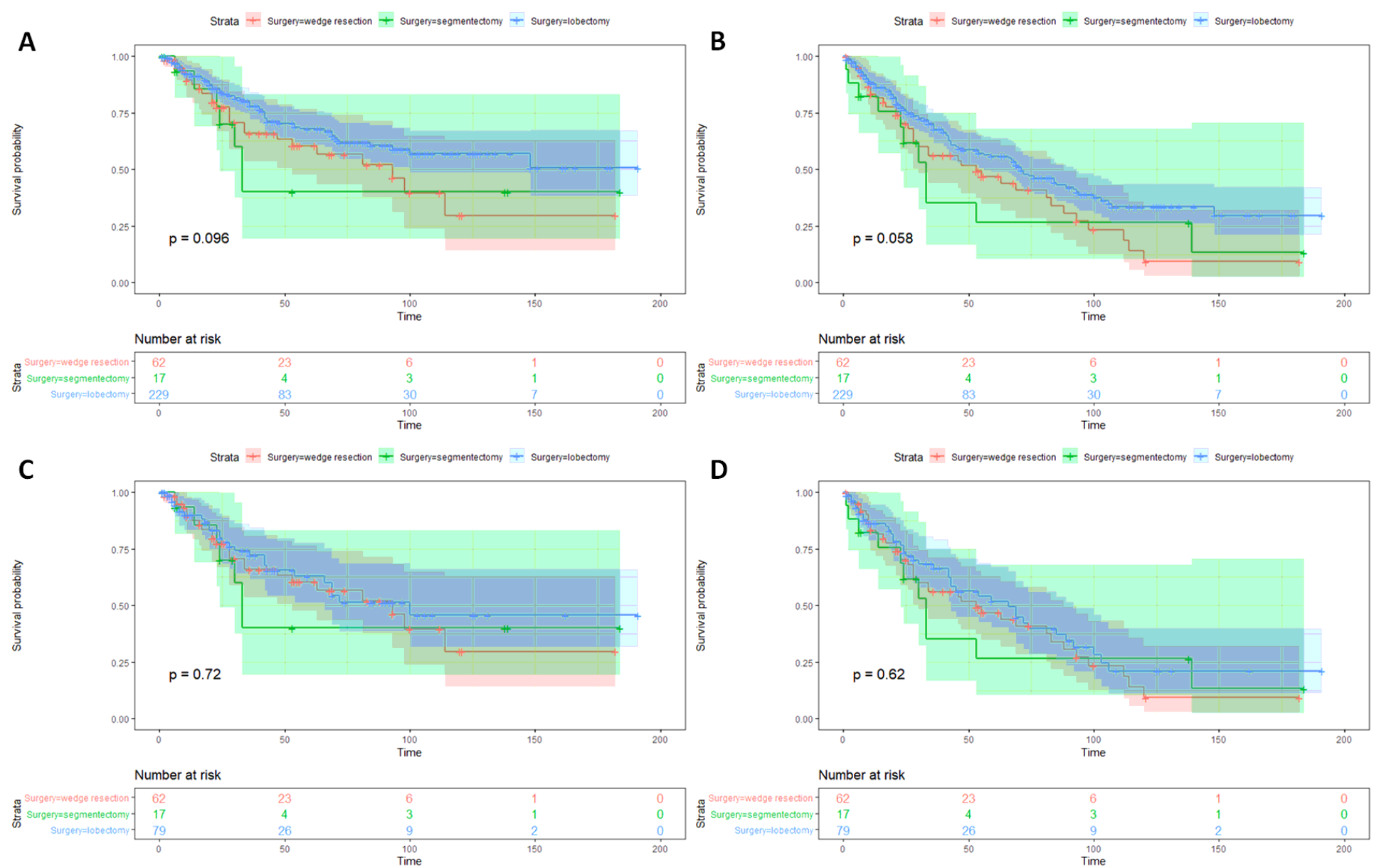

\section{Figure 2}

Kaplan-Meier survival curves of cancer-specific survival $(a, c)$ and overall survival $(b, d)$ between patients with wedge resection, segmentectomy and lobectomy before and after propensity score matching. 

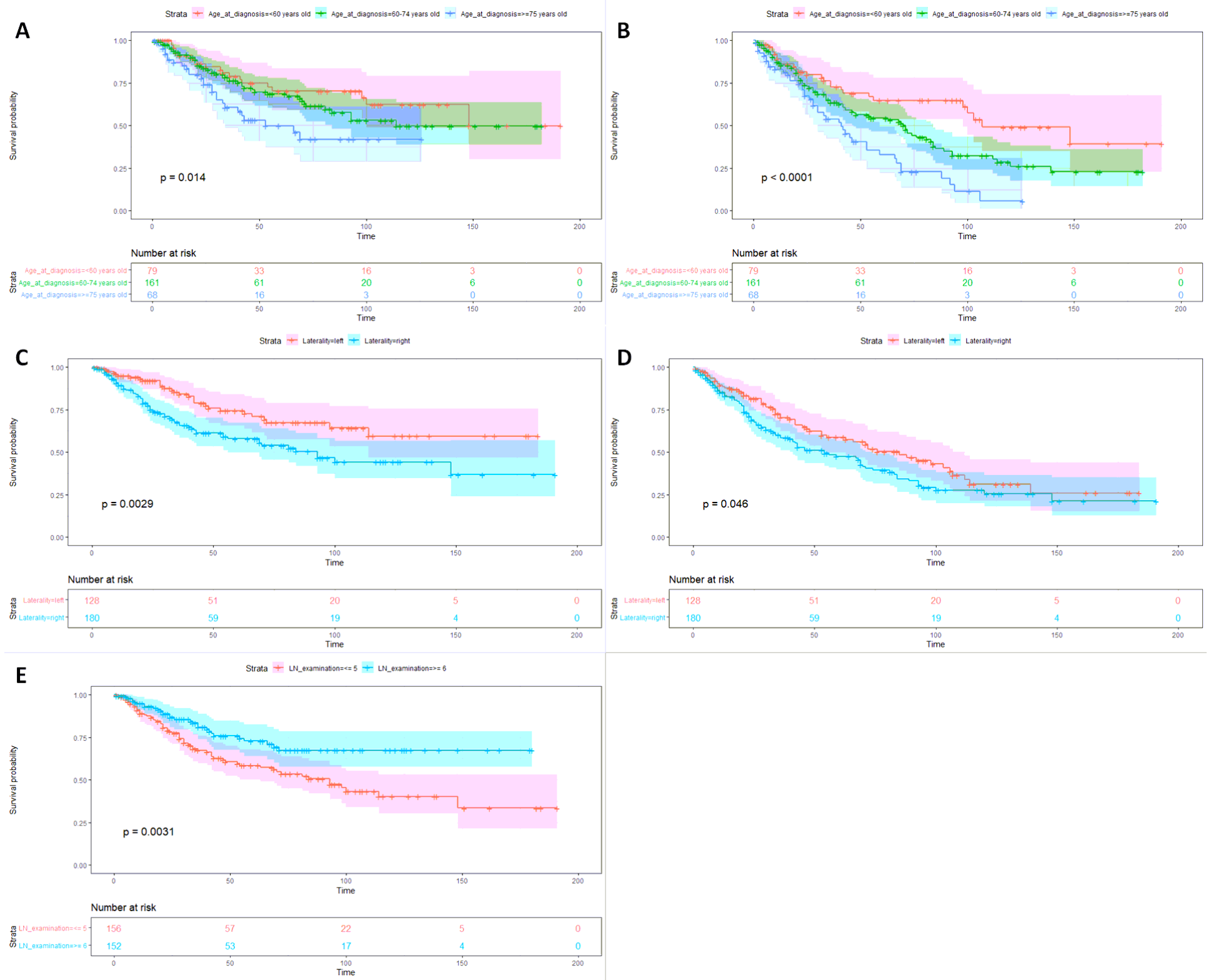

\section{Figure 3}

Kaplan-Meier survival curves of cancer-specific survival (a, c, e) and overall survival (b, d) based on age at diagnosis, laterality of tumor, and the number of lymph nodes dissection.

\section{Supplementary Files}

This is a list of supplementary files associated with this preprint. Click to download.

- Supplemental.docx 